\title{
Protocol for Sonospeech Cleft Pilot: A Pilot Randomised Control Trial of Ultrasound Visual Biofeedback Versus Standard Intervention for Children with Cleft Lip and Palate.
}

Joanne Cleland ( $\nabla$ joanne.cleland@strath.ac.uk)

University of Strathclyde https://orcid.org/0000-0002-0660-1646

Lisa Crampin

Queen Elizabeth University Hospital

Linsay Campbell

Queen Elizabeth University Hospital

Marie Dokovova

University of Strathclyde Faculty of Humanities and Social Sciences

Study Protocol

Keywords: Cleft lip and palate, articulation intervention, ultrasound visual biofeedback

Posted Date: September 30th, 2021

DOI: https://doi.org/10.21203/rs.3.rs-927181/v1

License: (c) (1) This work is licensed under a Creative Commons Attribution 4.0 International License.

Read Full License 


\section{Abstract}

Background: Children with cleft lip and palate can continue to have problems producing clear speech after surgery. This can lead to social, emotional, and educational challenges. Typical treatment involves teaching children the correct tongue movements to produce speech sounds. This is known as articulation intervention. However, this intervention is challenging because the tongue is hidden from view and movements are difficult to see and describe. This pilot randomized control trial will try a new treatment, ultrasound visual biofeedback (U-VBF).

Methods/Design: The Sonospeech project will enroll up to 40 children with cleft lip and palate aged 4;6 to 16 in a single-centre two-arm parallel group pilot randomized controlled trial with blinded assessors. Children will receive either six sessions of U-VBF or articulation intervention. The primary goals of this pilot are to determine recruitment/attrition rates; to measure pre-post follow up completion; and acceptability of the randomization and interventions to families.

Discussion: Larger trials of speech interventions for children with cleft lip and palate are needed. This pilot/feasibility study will determine whether a larger randomized control trial comparing ultrasound and articulation interventions is feasible.

Trial registration. ISRCTN, ISRCTN17441953. Registered 22 March 2021, http://isrctn.com/ISRCTN17441953. See Appendix 1 for all items.

\section{Background}

Cleft lip and palate (CLP) is the most common congenital craniofacial abnormality, occurring in one in 700 births [1]. Problems producing intelligible speech occur in CLP, even after surgery to repair the palate, and in some children cleft speech characteristics (CSC) persist requiring intervention from a speech and language therapist (SLT) [2]. This unclear speech has adverse social and educational consequences, with the speech of children with CLP rated as more likely to belong to someone who is "unhealthy", "no friends" and "ugly" [3]. Standard treatment is articulation intervention (Al). This approach involves teaching children correct placement for their articulators (primarily the tongue) through verbal description and demonstration [4]. Al is a challenging intervention for both the clinician and the patient because speech movements are both difficult to see and describe, due to the main articulator, the tongue, being largely hidden from view. This problem can be circumvented by using instrumental articulatory techniques which view and measure the articulators directly. Over the last few decades electropalatography (EPG) has dominated the literature as the instrumental technique of choice for people with cleft lip and palate. EPG measures tongue-palate contact using an artificial palate with electrodes embedded in it. This real-time dynamic image of tongue-palate contact can be used as a biofeedback tool to teach patients about correct placement of the tongue. However, a Cochrane review of EPG by Lee and colleagues [5] found that only one study met inclusion criteria. Despite a large number of studies using EPG, most were small $n$ or case studies. It is likely that larger studies using EPG are sparse 
because of practical issues with using this tool. Each patient requires an expensive custom-made palate, moreover, this custom-made palate only fits for a limited time period due to changing dentition and planned surgeries. In contrast, ultrasound visual biofeedback (U-VBF) is growing in popularity as biofeedback tool for children with speech disorders [6]. U-VBF holds several practical advantages over EPG: it is cheaper and does not require individualised equipment. Moreover, while EPG images only tongue-palate contact from the alveolar region to the boundary of the hard and soft palate, U-VBF images from near the tongue tip to the root, with pharyngeal articulations, common in CLP, clearly visible. This makes U-VBF arguably the technique of choice for CLP [7] yet it is relatively new to the Speech and Language Therapy clinic. This is because it is only now that ultrasound systems for measuring articulation provide fast enough frame rates at an affordable cost. A recent study showed that ultrasound can be used to identify all of the CSC described in the instrumental literature and that ultrasound assessment has better reliability than traditional perceptual approaches [8]. However, U-VBF has to our knowledge only be used in one small study with two participants with CLP [9]. The U-VBF treatment was effective for one of the children, aged 6;2, who reached $90 \%$ correct target productions and generalized to untrained words. His difficulties were with backing /k/ and fronting / $\nabla /$. The other child, aged 9;2, had only a modest increase from $5-21 \%$ of correct target consonants. His difficulties were with backing $/ \mathrm{n} /$ to palatal and velar position. In children with other types of speech sound disorders U-VBF shows positive outcomes for the majority of children and it is particularly useful for establishing new articulations [6]: an area of particular difficulty in CLP [4]. U-VBF is therefore potentially a useful tool for establishing new articulations in children with CLP. The aim of this study is therefore to assess the feasibility and inform the design of a full scale RCT of U-VBF for children with cleft speech characteristics.

\section{Methods/design}

\section{Aims}

The aim of this study is to assess the feasibility and inform the design of a full-scale RCT of U-VBF for children with cleft speech characteristics. The primary aim of U-VBF is to enable learning of new articulatory gestures (new speech sounds), with secondary aims of improving intelligibility and healthrelated quality of life.

\section{Design and Setting}

Mixed methods will be employed. The study is a single blind pilot randomised controlled trial, with control offered U-VBF therapy at the end of the study. A qualitative study (focus group) of the acceptability of both interventions and the study design will also be undertaken. All intervention will take place at the speech and language therapy hub in the Royal Hospital for Children in Glasgow. Eligibility screens and pre- and post-intervention assessments will take place either in person in a university clinic room or via telehealth (Zoom ${ }^{\mathrm{TM}}$ or Microsoft TEAMS ${ }^{\mathrm{TM}}$ ). The roles and responsibilities are outlined in Appendix 2. 


\section{Research questions}

No definitive comparisons of the interventions will be undertaken. The feasibility of a full scale RCT will be determined by evaluating a number of objectives against set success criteria (bulleted below) taken from a similar pilot RCT of children with speech disorders [10]:

Objectives:

1. To determine recruitment and attrition rates

- $75 \%$ of children and their families identified agree to participate

- $75 \%$ of children allocated in each group are retained for the duration of the study

2. To measure pre-post and follow-up outcome measure completion

- $75 \%$ of outcome measures are completed

3. To measure within-session outcome measure completion

- Data is reported from $75 \%$ of intervention sessions

4. To determine acceptability of randomisation to children and their families

- $75 \%$ of children and their families rate randomisation as acceptable in a questionnaire

5. To determine the acceptability of ultrasound visual biofeedback as an assessment tool (both groups) and intervention tool (U-VBF group)

- $75 \%$ of children and their families rate ultrasound as an acceptable technique in a questionnaire.

- Focus group analysis contains more positive than negative themes regarding acceptability

6. To measure adherence to the treatment protocol

- $75 \%$ of sessions reach the minimum dosage of 100 trials in both treatment arms

\section{Methods}

A single-centre two-arm parallel group pilot randomised controlled trial with blinded assessors will be carried out. Cases will be stratified by three age groups $(4 ; 6-7 ; 11 ; 8 ; 0-11 ; 11$ and $12 ; 0-16 ; 0)$. Due to the nature of the U-VBF, therapists and patients will not be blinded to treatment allocation but the limitations of this will be mitigated by the use of an assessor, blinded to group, and evaluators, blinded to both group and treatment time point. Unblinding to the assessor or the evaluator will not be permissible and is considered unnecessary given the clinicians delivering the intervention are not blind to treatment allocation. 


\section{Participants}

Children managed by the Scottish Cleft Lip and Palate service aged 4;6 to 16 will be identified and recruited when attending SLT clinics at the Royal Hospital for Children in Glasgow. Invitation letters containing the complete study information sheets will be sent to families. Inclusion criteria regarding age and cleft-type are broad to reflect current clinical practice, however, inclusion criteria for type of cleft speech characteristic are narrower to ensure children are likely to benefit from either U-VBF or the control intervention. Children are eligible if they have at least one speech error that would normally be a candidate for articulation intervention. We aim to recruit 20 children to each arm of the trial. Recruitment will stop once the target number of participants is recruited and within two years from the beginning of the study. No interim analyses will be performed before all data is collected. Children with an uncorrected bilateral hearing loss of greater than $30 \mathrm{~dB}$ (from previous reports), planned surgery within the next three months, or severe language deficit (from previous SLT reports and a receptive vocabulary standard score $<70$ on the BPVS-3, [11] ) will be excluded. Children will be allowed to continue all other medical and speech and language interventions during the trial. We will collect information on any other speech and language interventions received during the trial.

Following baseline, the children will be randomised by the Glasgow Clinical Trials Unit in a 1:1 ratio, stratified for age. Children randomised to the control arm will be offered U-VBF at the end of the trial if they still present with CSCs which are candidates for U-VBF as previous studies have shown this improves the acceptability of a randomised trial to families [10].

\section{Eligibility/Baseline Assessments:}

We will screen potential participants from case-notes and invite them to attend an initial screening and baseline assessment. The person carrying it out will obtain consent, ensuring the participants and their families have had time to read, understand and discuss the information about the study, prior to any assessment or procedures. This assessment will be either in person or via video conferencing, with inperson preferred. Screening assessment will comprise the British Picture Vocabulary Test 3 [11] to screen for adequate receptive vocabulary; and a speech assessment protocol to determine whether patients present with at least one cleft speech characteristic which would be amenable to both U-VBF and the control intervention. This assessment protocol comprises the Diagnostic Evaluation of Articulation and Phonology [12] articulation and phonology subtests and an ultrasound tongue imaging protocol designed in a previous project [8] to identify covert speech errors from consonants at all places of articulation and sentences from the GoS.SP.aSS.'98/ CAPS-A [13] (Appendix 1). Families who opt for the assessment over video-conferencing will complete the same assessments, but the ultrasound tongue imaging protocol will be replaced with a audio-perceptual assessment of the same materials[1].

\section{Speech Target Selection}


Children with CLP may present with multiple CSCs affecting intelligibility. We intend to select as intervention targets speech sounds which are 1. amenable to treatment with both interventions and 2 . likely to have the biggest functional impact on intelligibility. Following the screening assessments, we will select wordlists targeting each child's specific lingual errors from a battery. In English, lingual speech sounds (imageable with ultrasound and amenable to treatment with both interventions) are

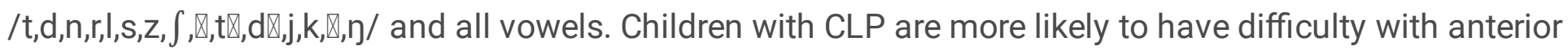
consonants $/ t, d, s /$. Wordlists containing these speech sounds will also form a key outcome measure (see below). Two wordlists per error type will be selected, firstly an "untreated probe" (i.e., the words will not be used in the course of therapy, this allows us to check for generalisation of targets). Secondly a "treated probe", containing high frequency and functionally useful words will be used to train speech targets in the course of therapy. The wordlists contain lingual targets in increasingly complex contexts from single syllable words/pseudowords through to multi-syllabic words and sentences. Where the child has more than one error, multiple wordlists will be used, however only one treatment target (the speech sound with the most errors) will be selected and the other errors will serve as "control segments" (i.e., speech sounds that should not improve during the course of therapy unless maturation is a factor). Wordlists will be analysed for percentage target consonants correct (PTC). Children must score $<30$ PTC at baseline to be eligible for the study.

\section{Interventions}

Both interventions will be delivered by the cleft palate specialist SLTs in the Royal Hospital for Children in Glasgow. Therapy in both treatment arms will be once per week for six sessions with each session lasting up to $45 \mathrm{mins}$. The number of sessions is pragmatic in nature, reflecting current practice, and is designed to highlight initial response to both interventions. It is likely that some children in both arms of the trial may require further speech intervention in the future (after the follow-up measures are taken) and this will be provided in line with standard practice, which includes further U-VBF. Both interventions will focus on acquisition of new speech sounds. Previous studies of children with non-cleft speech disorders show that a new speech sound can be acquired within one to two sessions [14] of U-VBF for most children but that some children take four to six sessions. Target articulations will be decided individually. Both interventions begin with a pre-practice phase where the aim is to teach the child to approximate the target articulation before they can begin the practice phase where at least 100 repetitions are required for learning and generalisation. This dosage will be measured in both interventions. In this pilot we will focus on both the pre-practice phase and the first stage of practice: acquisition of a new sound in simple contexts such as "ta, tea, toe" building to short words such as "tap, team, tore" as this is feasible within six intervention sessions. If children are super-responders (i.e., they quickly retain the new speech sound and are able to produce it in complex contexts) then the protocol will also allow us to measure this. Participants will be discontinued from the intervention if they show any adverse effects to the ultrasound treatment- in rare cases the ultrasound gel can cause contact dermatitis [15] 


\section{Articulation Intervention (Al)}

This intervention involves working on a single speech sound at a time. The SLT uses modelling, demonstration, verbal description, and feedback in the pre-practice phase to teach the child the new sound at first in limited contexts and then in words and finally in conversation in the practice phase. To increase parity with U-VBF, and in line with newer theories of motor learning [16], we will standardise the in-session dosage during the practice phase to at least 100 trials, i.e. each child will be given 100 attempts to articulate their target articulation in each session.

\section{Ultrasound Visual Biofeedback (U-VBF)}

This intervention is grounded in the principles of motor learning. The patient sees a real-time image of their tongue moving and guided by the SLT uses this biofeedback to learn a new articulation, building productions to increasingly complex contexts, as in Al. Again, a minimum of 100 trials are required in the intervention. The intervention is set out in an open access manual [17] and involves using the software Sonospeech ${ }^{\mathrm{TM}}$. The software has functionality to be used as an assessment and intervention tool, allowing the SLT and patient to record and playback ultrasound video with synchronised audio or to view it live. The clinicians delivering the intervention have completed training and currently use ultrasound in their clinical practice.

\section{Outcome Measures}

A blinded assessor will collect measures at baseline (-t1: pre-treatment), 2 months post-randomization ( $\mathrm{t}$, to allow for any delays in referral to therapy), and 3 months post-randomization (t8) to see if any benefit is maintained. Assessment will take place in a university clinic or via video-conferencing and will be carried out by a research SLT blinded to group for primary outcome measures and by the treating SLTs for within-session measures. See table 1.

\section{Within-treatment session outcomes}

Previous research suggests that one of the main benefits of U-VBF may be efficiency rather than overall efficacy of treatment [6]. We will therefore measure treatment response during each session, rather than just after the course of treatment. The treating SLTs will therefore audio record short treated, and untreated word lists at every treatment session (t1-t6). From this we will determine how quickly children achieve a new articulation as a measure of response to treatment. These will be rated at the end of the project by SLTs blinded to group.

\section{Candidate Primary Outcome Measures}


The key primary outcome linear measure for change in speech will be percentage target consonants correct (PTC). We will measure this at single sound level (stimulability and in /aCa/ contexts); single word level; and sentence level in treated and untreated wordlists (see above section on speech target selection). All direct speech measures will be recorded with audio and where possible ultrasound tongue imaging in both groups, allowing us to perform ultrasound analysis of data from both groups.

\section{Patient Reported Outcome Measures}

We will use the Intelligibility in Context Scale [18] as a carer reported outcome. This short scale asks parents/carers to rate how easy to understand their child is to a variety of listeners ranging from family members to strangers. It has been shown to have a high internal reliability and construct validity [18]. Quality of life will be measured using the CLEFT-Q speech function and quality of life scales for children aged 8 and over [19]. This instrument has good content and construct validity, and good reliability, as established using a large and diverse international sample of participants [19]. We will also use the Experience of Service Questionnaire [20] to measure patient and carer satisfaction with both interventions at the end of the project. It was shown to have good construct validity and precision in measuring satisfaction with care [20]. The bespoke data collection forms, such as the participant information sheets and the consent form templates, can be found in a publicly accessible folder on the University of Strathclyde open access Knowledge Base "PURE".

\section{Intervention Acceptability Measures and Qualitative Evaluation}

Families will complete a questionnaire about the acceptability of both interventions at the end of the study. These questionnaires will also be offered to families of participants who drop out of the study. Participant retention will be promoted by dedicating time to discuss any questions and problems that may have arisen for the participant and their family at the start of each session and by offering further ultrasound or articulation intervention after the final follow-up if indicated. Parents/carers and children over 12 will be invited to join focus groups to discuss their experiences of taking part in the trial and to contribute to planning a larger trial. Each focus group will include up to 10 participants. Responses to the focus groups will be analysed using thematic analysis.

Table 1 shows the timeline for the project, including the timing of each assessment. 


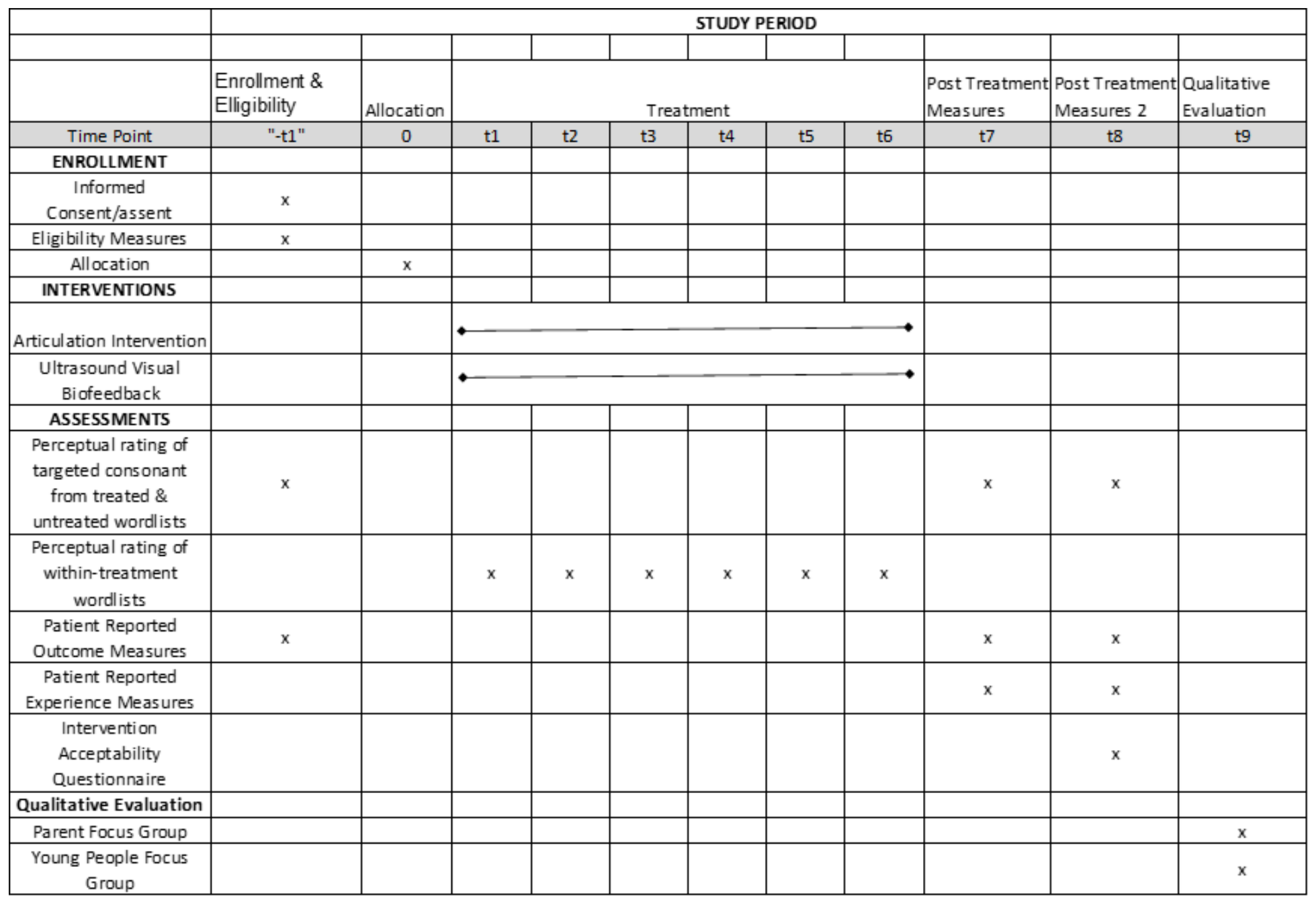

\section{Analyses and Statistical Power}

Definitive comparisons of the interventions will not be undertaken due to the feasibility nature of the study. Details of patient screening, recruitment, retention, withdrawal, follow-up will be summarised (see research questions above). Adherence to U-VBF will be measured according to the number of patients who complete the intervention in accordance with the treatment manual. Adherence to treatment dosage will be recorded using an intervention pro-forma in each session where the SLT records a tick mark for each trial (i.e., each time the patient attempts to produce the speech sound in treatment, this should be around 100 for both interventions). All sessions will be audio-recorded (consent permitting) and $20 \%$ of sessions will be fidelity checked for dosage and adherence to protocol.

\section{Ultrasound/Speech Analysis}

All of the speech measures at baseline, and follow-up will be recorded with simultaneous ultrasound in both groups by a research SLT blinded to group where possible. Our previous work showed that the addition of ultrasound to transcription increases inter-rater reliability and allows identification of covert (imperceptible to the ear alone) errors [9]. This will allow us both to calculate PTC (the primary outcome 
measure) with increased reliability and to perform an error analysis. $25 \%$ of the data will also be rated by two specialist cleft SLTs (not involved in the project) trained in ultrasound-aided transcription. These SLTs will also rate the audio recordings from the within-treatment sessions ( 11 to t6 in Table 1), blinded to group.

\section{Data management}

All data will be stored in a dedicated secure shared drive managed by the University of Strathclyde where it will be automatically backed up. Hard copies of data will be kept at the Hospital and the University of Strathclyde. Participants will be pseudo-anonymised during the project, with a key linking codes to actual names held securely at the University. The key will be deleted at the end of the project and data will become anonymous. Participants and their families will be informed that recordings of voice can be identifiable.

\section{Harms}

Based on previous studies using ultrasound intervention for speech sound disorders, no harm to the participants is anticipated. In rare cases, some people may have an allergic reaction (contact dermatitis) to the gel [15]. If this is observed the gel can be substituted with water. If water does not achieve a sufficiently good ultrasound image, then the participant may be discontinued from the trial. If any adverse consequences are observed, they will be recorded in the participant's clinical notes and reported to the NHS ethics committee using standard procedures. The ethics committee and the University ethics committee can audit the trial at any time.

\section{Dissemination policy}

The results from this study will be published in academic journals, such as The Cleft Palate Craniofacial Journal, Clinical Linguistics and Phonetics. Additionally, results will be presented at academic and clinical conferences such as the International Congress of Cleft, Lip and Palate and Related Craniofacial Anomalies, and the conference of the Royal College of Speech and Language Therapists, where it will reach both an international and a local audience of clinicians.

The protocol, statistical code and the anonymized final numerical dataset will be made available publicly on the University of Strathclyde's open access repository "PURE". Protocol changes will be reported to the trial registry and the ethics committee. 
[1] The assessment sessions are not part of core clinical care and therefore we will offer these over videoconferencing to comply with any current local Covid restrictions under which travelling for research assessments may not be an "essential journey".

\section{Discussion And Summary}

This pilot feasibility study will determine whether a full-scale randomised control trial comparing ultrasound visual biofeedback to treatment as usual (articulation intervention) for the treatment of cleft speech characteristics is feasible. Prior studies such as $[8,14]$ suggest that children with CLP can tolerate ultrasound recordings and studies such as [14] suggest good retention in ultrasound intervention, with only one child in this study lost to follow-up. We therefore predict that the objectives outlined above will be met, and if this is the case a full-scale, adequately powered, multi-centre RCT will be warranted. Within this planned larger RCT we hypothesise that U-VBF will show quicker acquisition of speech sounds in error, leading to shorter time in intervention, reduced frustration for patients, and cost savings. Nevertheless, there are some potential limitations to the current study. Firstly, it is not known how acceptable randomisation will be to patients and carers and it is possible that carers will express a preference for the trial intervention, given the novelty of the technology used in U-VBF [23]. To mitigate this, patients will be offered U-VBF at the completion of the study, if their speech errors remain. A previous study trialing a similar technology-based intervention with children with Down syndrome showed that no children were lost to follow-up in either arm of the trial when this was offered [21]. A further limitation of the current study, which cannot be completely mitigated, is a potential preference from the treating clinicians for one intervention over the other, in particular the trial intervention. This could lead to increased outcomes in the trial intervention, which are not due to the visual biofeedback per se, but more to increased motivation in the intervention sessions [22]. Finally, a recent move towards more use of telehealth due to the Covid-19 pandemic, means that U-VBF is disadvantaged compared to articulation intervention, because it is a behavioural intervention which requires hardware and therefore only be can only be carried out in person, rather than over video-conferencing. It therefore currently may be more difficult to recruit to a trial which involves travel to a hospital or university.

In conclusion, this feasibility study will determine whether a larger trial comparing U-VBF and Articulation Intervention is both feasible and acceptable to patients and their carers. Notwithstanding the limitations, we predict that achieving the success objectives related to recruitment, retention, and acceptability outlined above will motivate a full scale RCT which in turn seeks to determine whether U-VBF shows improved outcomes over treatment as usual for speech disorders associated with cleft lip and palate.

\section{Declarations}

\section{Ethics approval and consent to participate}

The study has been granted ethics approval by the West Midlands - South Birmingham Research Ethics Committee, reference number 21/WM/0104. 


\section{Consent for publication}

The manuscript does not contain any individual person's data in any form.

\section{Availability of data and materials}

All data underpinning this publication are openly available from the University of Strathclyde PURE Knowledge Base at: https://doi.org/10.15129/f65343c4-7781-44fe-9b00-516d4597efac

\section{Competing interests}

The authors declare that they have no competing interests.

\section{Funding}

The study is funded by the Chief Scientist Office of Scotland, Grant Number TCS/20/02. The funding body and the sponsor have no role in the design of the study and collection, analysis, and interpretation of data and in writing the manuscript.

\section{Acknowledgements}

Not applicable.

\section{Authors' contributions}

All authors contributed to the design of this protocol and have approved the submitted version. All authors have agreed both to be personally accountable for the author's own contributions and to ensure that questions related to the accuracy or integrity of any part of the work, even ones in which the author was not personally involved, are appropriately investigated, resolved, and the resolution documented in the literature.

\section{Authors' information}

School of Psychological Sciences and Health, University of Strathclyde

Joanne Cleland

Marie Dokovova

The Royal Hospital for Children in Glasgow, NHS Greater Glasgow and Clyde

Lisa Crampin

Linsay Campbell

\section{References}


1. Bellis TH, Wohlgemuth B: The Incidence of Cleft Lip and Palate Deformities in the South-east of Scotland (1971-1990). British Journal of Orthodontics 1999, 26(2):121-125.

2. Medina J, Fitzsimons, K., Deacon, S., Russell, C., Wahedally, H., Park, M.H., van der Meulen, J.: Cleft Registry and Audit Network Annual Report. In.: The Clinical Effectiveness Unit at the Royal College of Surgeons of England; 2019: 1079.

3. Lee A, Gibbon FE, Spivey K: Children's attitudes toward peers with unintelligible speech associated with cleft lip and/or palate. The Cleft Palate-Craniofacial Journal 2017, 54(3):262-268.

4. Bessell A, Sell D, Whiting P, Roulstone S, Albery L, Persson M, Verhoeven A, Burke M, Ness AR: Speech and language therapy interventions for children with cleft palate: a systematic review. The Cleft Palate-Craniofacial Journal 2013, 50(1):1-17.

5. Lee ASY, Law J, Gibbon FE: Electropalatography for articulation disorders associated with cleft palate. Cochrane Database of Systematic Reviews 2009.

6. Sugden E, Lloyd S, Lam J, Cleland J: Systematic review of ultrasound visual biofeedback in intervention for speech sound disorders. International journal of language \& communication disorders 2019.

7. Bressmann T, Radovanovic B, Kulkarni GV, Klaiman P, Fisher D: An ultrasonographic investigation of cleft-type compensatory articulations of voiceless velar stops. Clinical linguistics \& phonetics 2011, 25(11-12):1028-1033.

8. Cleland J, Lloyd S, Campbell L, Crampin L, Palo J-P, Sugden E, Wrench A, Zharkova N: The impact of real-time articulatory information on phonetic transcription: ultrasound-aided transcription in cleft lip and palate speech. Folia Phoniatrica et Logopaedica 2019:1-11.

9. Roxburgh Z, Cleland J, Scobbie JM: Multiple phonetically trained-listener comparisons of speech before and after articulatory intervention in two children with repaired submucous cleft palate. Clinical Linguistics \& Phonetics 2016, 30(3-5):398-415.

10. Pennington L, Stamp E, Smith J, Kelly H, Parker N, Stockwell K, Aluko P, Othman M, Brittain K, Vale L: Internet delivery of intensive speech and language therapy for children with cerebral palsy: a pilot randomised controlled trial. BMJ Open 2019, 9(1):e024233.

11. L. Dunn DD, J. Sewell and B. Styles: British Picture Vocabulary Scale 3. Swindon: GL Assessment 1997.

12. Dodd B, Zhu H, Crosbie S, Holm A, Ozanne A: Diagnostic evaluation of articulation and phonology (DEAP): Psychology Corporation; 2002.

13. Sell D, John A, Harding-Bell A, Sweeney T, Hegarty F, Freeman J: Cleft Audit Protocol for Speech (CAPS-A): a comprehensive training package for speech analysis. International journal of language \& communication disorders 2009, 44(4):529-548.

14. Cleland J, Scobbie JM, Roxburgh Z, Heyde C, Wrench A: Enabling new articulatory gestures in children with persistent speech sound disorders using ultrasound visual biofeedback. Journal of Speech, Language, and Hearing Research 2019, 62(2):229-246. 
15. Chasset F, Soria A, Moguelet P, Mathian A, Auger Y, Francès C, Barete S: Contact dermatitis due to ultrasound gel: A case report and published work review. The Journal of Dermatology 2016, 43(3):318-320.

16. Ruscello D, Vallino L: The Application of Motor Learning Concepts to the Treatment of Children with Compensatory Speech Sound Errors. Perspectives on Speech Science and Orofacial Disorders 2014, 24(2):39-47.

17. Cleland J, Wrench A, Lloyd S, Sugden E: ULTRAX2020: Ultrasound Technology for Optimising the Treatment of Speech Disorders: Clinicians' Resource Manual. 2018.

18. McLeod S, Harrison LJ, McCormack J: The intelligibility in context scale: Validity and reliability of a subjective rating measure. Journal of Speech, Language, and Hearing Research 2012.

19. Klassen AF, Riff KWW, Longmire NM, Albert A, Allen GC, Aydin MA, Baker SB, Cano SJ, Chan AJ, Courtemanche DJ: Psychometric findings and normative values for the CLEFT-Q based on 2434 children and young adult patients with cleft lip and/or palate from 12 countries. CMAJ 2018, 190(15):E455-E462.

20. Brown A, Ford T, Deighton J, Wolpert M: Satisfaction in child and adolescent mental health services: Translating users' feedback into measurement. Administration and Policy in Mental Health and Mental Health Services Research 2014, 41(4):434-446.

21. Wood SE, Timmins C, Wishart J, Hardcastle WJ, Cleland J: Use of electropalatography in the treatment of speech disorders in children with Down syndrome: a randomized controlled trial. International Journal of Language \& Communication Disorders 2019, 54(2):234-248.

22. McAllister T, Preston JL, Hitchcock ER, Hill J: Protocol for Correcting Residual Errors with Spectral, ULtrasound, Traditional Speech therapy Randomized Controlled Trial (C-RESULTS RCT). BMC Pediatrics 2020, 20(1).

\section{Supplementary Files}

This is a list of supplementary files associated with this preprint. Click to download.

- Appendix.docx

- SPIRIT2013Checklist.docx 